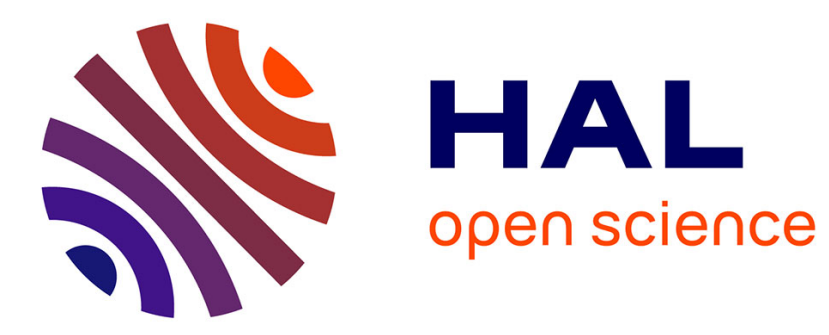

\title{
Modeling cooperative and autonomous adaptive cruise control dynamic responses using experimental data
}

\author{
Vicente Milanés, Steven E. Shladover
}

\section{To cite this version:}

Vicente Milanés, Steven E. Shladover. Modeling cooperative and autonomous adaptive cruise control dynamic responses using experimental data. Transportation research. Part C, Emerging technologies, 2014, pp.285-300. 10.1016/j.trc.2014.09.001 . hal-01091160

\section{HAL Id: hal-01091160 https://hal.science/hal-01091160}

Submitted on 5 Dec 2014

HAL is a multi-disciplinary open access archive for the deposit and dissemination of scientific research documents, whether they are published or not. The documents may come from teaching and research institutions in France or abroad, or from public or private research centers.
L'archive ouverte pluridisciplinaire HAL, est destinée au dépôt et à la diffusion de documents scientifiques de niveau recherche, publiés ou non, émanant des établissements d'enseignement et de recherche français ou étrangers, des laboratoires publics ou privés. 


\title{
Modeling Cooperative and Autonomous Adaptive Cruise Control Dynamic Responses Using Experimental Data
}

\author{
Vicente Milanés, Steven E. Shladover \\ California PATH Program of the Institute of Transportation Studies, University of \\ California, Richmond, CA 94804 e-mail: vicente.milanes@berkeley.edu, \\ steve@path.berkeley.edu
}

\begin{abstract}
Vehicle longitudinal control systems such as (commercially available) autonomous Adaptive Cruise Control (ACC) and its more sophisticated variant Cooperative ACC (CACC) could potentially have significant impacts on traffic flow. Accurate models of the dynamic responses of both of these systems are needed to produce realistic predictions of their effects on highway capacity and traffic flow dynamics. This paper describes the development of models of both ACC and CACC control systems that are based on real experimental data. To this end, four production vehicles were equipped with a commercial ACC system and a newly developed CACC controller. The Intelligent Driver Model (IDM) that has been widely used for ACC car-following modeling was also implemented on the production vehicles. These controllers were tested in different traffic situations in order to measure the actual responses of the vehicles. Test results indicate that: 1) the IDM controller when implemented in our experimental test vehicles does not perceptibly follow the speed changes of the preceding vehicle; 2) consecutive strings of ACC vehicles are unstable, amplifying the speed variations of preceding vehicles; and 3) consecutive strings of CACC vehicles overcome these limitations, providing smooth and stable car following responses. Simple but accurate models of the ACC and CACC vehicle following dynamics were derived from the actual measured responses of the vehicles and applied to simulations of some simple multi-vehicle car following scenarios.
\end{abstract}

Keywords: Cooperative Adaptive Cruise Control (CACC), Adaptive Cruise Control (ACC), car-following models, vehicle platooning, intelligent 
transportation systems (ITS)

\section{Introduction}

Freeway congestion in the vicinity of large cities increases daily pollution and fuel consumption, and wastes commuters time. Data indicate that U.S. commuters experienced an average of 52 hours of delay per year, causing $\$ 121$ billion of delay and fuel costs annually [20]. This problem is being approached in two different ways. On one hand, some projects work on the infrastructure side, trying to provide information about the road traffic conditions and advising drivers on travel times to permit rerouting, as well as managing the flows of vehicles onto highway on-ramps. On the other hand, other projects focus on the vehicles, developing intelligent systems able to adjust vehicles speeds based on the preceding vehicles speeds, modifying the dynamics of the traffic response [33].

The first commercially available systems that actively controlled vehicle speeds independent of driver actions were Conventional Cruise Control (CCC) systems, based on regulating the throttle action to control vehicle speed [23]. The next step in the development of speed control systems was the Adaptive Cruise Control (ACC) [22]. As occurs with CCC, a set speed can be chosen using the driver interface but when a preceding vehicle is detected by a lidar or radar sensor, the subject vehicle speed is adapted to the preceding one based on a pre-selected time gap. The driver's tasks are considerably reduced under regular traffic circumstances because control of the distance to the preceding vehicle, via both brake and throttle action, is done automatically [34].

ACC systems are currently available in the market, primarily on premium vehicles, but with some introductions on middle-class vehicles. The design and control strategies of the commercially available ACC systems are closelyguarded secrets of their developers because these are sensitive to competition between auto makers and suppliers. The open literature only contains documentation of similar systems developed in academic research projects, only a few of which have been experimentally implemented on real vehicles. A literature review shows a variety of technical approaches. Among them, an explicit model predictive control approach is presented in [15]. [13] shows a full-range speed ACC with collision avoidance capabilities including different driving modes as a function of the vehicle's acceleration. Control based 
on artificial intelligence techniques for ACC is presented in [14]. In [12], a comparative study between robust control approach and artificial intelligence based control is shown for a low speed range ACC.

The next generation of longitudinal control systems is focused on adding vehicle-to-vehicle $(\mathrm{V} 2 \mathrm{~V})$ communications to take advantage of more extensive preceding vehicle information, from multiple preceding vehicles, and with reduced delays. Using this additional information, control system performance can be tightened significantly, enabling significant reductions in inter-vehicle distance and improvements in string stability [25]. Recently, its potential benefits were tested in the Grand Cooperative Driving Challenge held in Helmond, The Netherlands in 2011[32] where nine vehicles from different European research institutions drove in a two-lane platoon using different control techniques mainly based on proportional, or proportional-derivative feedback/feedforward controllers [9, 17, 4] or Model Predictive Control (MPC) techniques $[3,8]$. A successful demonstration was also carried out by The Connect \& Drive project using a six-vehicle platoon [18] in The Netherlands. Finally, the first PATH vehicle following results using a two-vehicle CACC implementation were presented in [2].

Impacts of ACC and CACC systems on traffic flow have been widely analyzed through simulation studies, assuming a range of market penetrations. Specifically related to ACC, [1] presented an extensive review about different car-following models for traffic analysis. Recently, the Intelligent Driver Model (IDM) [29] has been used as a reference for modeling ACC car-following behavior in traffic flow simulations. It defines the target acceleration for the follower vehicles using a free-road strategy for the acceleration phase and a combined strategy of the desired time gap and comfortable deceleration for the braking phase. This approach has been enhanced [6] to avoid hard braking responses in case of cut-in maneuvers, in which a new vehicle merges into the lane. Recently, the IIDM model has also been introduced [30], including modifications to the original IDM model for situations where actual speed exceeds the desired speed and/or is close to it. Highway capacity simulations using this car-following logic have shown predictions of capacity improvement even with a low ACC market penetration (under 50\%). The General Motors car-following model is used in [5] where highway capacity drastically increases when the percentage of ACC-equipped vehicles approaches $100 \%$. However, when the ACC market penetration is high, a small increase in the percentage of human drivers was found to be enough to cause congestion. An analytical study of mixed traffic flow between human-driven vehicles and 
IDM-equipped vehicles is shown in [16] considering time-delay response in both.

Regarding CACC results, [31] presented CACC impacts on merging situations on highways when the number of lanes is reduced from four to three. A new car-following model for CACC vehicles was introduced and the results show CACC dramatically improving traffic flow with a reasonable increment in the market penetration. Lately, [19] introduced a modified version of the IDM controller (IDM+) for studying traffic flow stability. Experimental results based on a 50-vehicle platoon where recommended acceleration or braking commands were suggested to the driver were also shown. It was tested with a $100 \%$ market penetration rate but it significantly reduced shockwaves. A simple model for CACC and ACC vehicles is presented in [26] where results showed that the increase in the number of ACC vehicles is unlikely to produce any significant change in highway capacity. However, when the CACC-equipped vehicles are over $50 \%$ market penetration, lane capacity is drastically increased. Although a limited number of results for both ACC and CACC implemented on production vehicles are available, models used for macroscopic simulations have been based on theoretical approaches rather than on experimental results. That is, there has until now been no direct relationship between the results obtained on production vehicles and the simulations carried out to evaluate ACC and CACC traffic impact.

In this review, we focus on ACC and CACC designs that are based on constant time-gap (sometimes loosely referred to as constant-headway) vehicle following criteria, which are most closely related to normal manual vehicle-following behavior by drivers. These are substantially different from the constant-clearance (or constant-spacing) vehicle following criteria used in tightly coupled platooning concepts that have been developed and tested for cars and trucks. The control design and stabilization challenges are significantly more difficult for the latter class of systems, as explained by [27].

This paper first analyzes two consecutive vehicles using a commercial ACC system, the CACC controller developed by the project team [11] and the IDM controller, all implemented on the same production cars. Next, experimental results for four consecutive vehicles using both the commercial ACC controller and the CACC controller are presented. From these results, a new model for ACC and CACC controller dynamic responses is identified, to be used for microscopic simulation purpose. Simulation results for both controllers are included and compared with the experimental results obtained from the production vehicles. Results indicate that while CACC is 
likely to improve traffic flow, ACC can cause bigger traffic jams than human drivers, contrary to the large majority of the previous results presented in the literature. This idea was introduced in [26].

The rest of the paper is structured as follow. Section 2 introduces the production vehicles used in the tests and the comparison between the commercial ACC controller, the newly developed CACC system and the implementation of the IDM model for two consecutive vehicles. A test of four consecutive vehicles with both ACC and CACC controllers is presented in Section 3, where vehicle responses are analyzed. ACC and CACC vehicle models are proposed in Section 4. Section 5 presents some example simulation results to predict $\mathrm{ACC}$ and $\mathrm{CACC}$ car-following behavior when the number of consecutive equipped vehicles increases. Final remarks are given in Section 6.

\section{Test vehicle description}

Experiments were carried out using up to four production Infiniti M56s (see Figure 1) provided by Nissan. For the experimental test purposes, neither control input saturations nor gear changes were involved, producing a homogeneous vehicle chain. They are equipped with a commercial ACC system that uses a lidar for detecting the preceding vehicle. These vehicles have been retrofitted with a $5.9 \mathrm{GHz}$ Dedicated Short Range Communication (DSRC) system for permitting vehicle-to-vehicle (V2V) communications. A Differential Global Positioning System (DGPS) with Wide Area Augmentation System (WAAS) corrections is incorporated in a Wireless Safety Unit (WSU) supplied by DENSO. The control logic is embedded in a dSpace MicroAutoBox which receives data from both the WSU (i.e., preceding vehicle speed measurements from its on-board sensors, speed reference when controller is activated, controller activation flag, current time gap and set speed) and from the subject vehicle's Controller Area Network (CAN) (i.e., inter-vehicle distance measurements from the lidar).

Low-level commands-i.e., throttle and brake pedal actions-are generated by the on-vehicle production system. The high level controller (ACC or CACC) is in charge of generating speed commands to the low-level controller and these can be computed to modify the vehicle speed command using dSpace. Internal limitations are also imposed on the maximum accelerationup to $1 \mathrm{~m} / \mathrm{s}^{2}$-and deceleration-up to $2.8 \mathrm{~m} / \mathrm{s}^{2}$. 


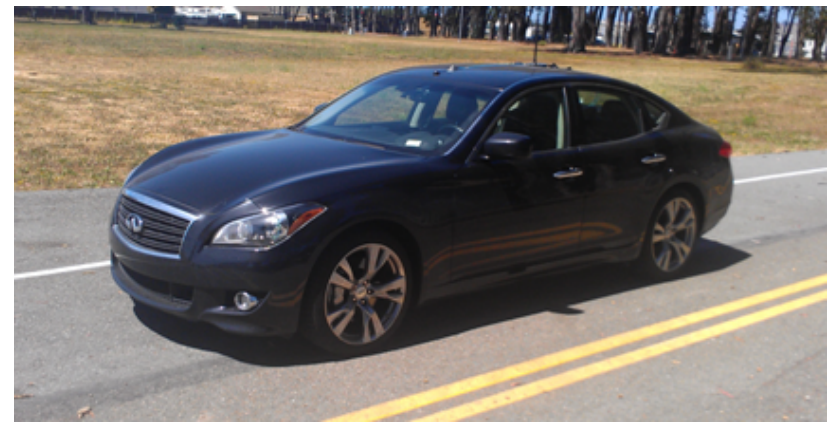

Figure 1: Infiniti M56s test vehicle equipped with DSRC communications

\subsection{Two-vehicle experimental results}

An on-road test with the described experimental platform was carried out using the different controllers to evaluate their responses in real traffic conditions. The tested controllers were: 1) the factory equipped ACC system; 2) the CACC controller that has been previously developed and presented in [11]; and 3) the IDM model that has been widely used as a reference for ACC car-following models. A two-vehicle comparative study was carried out considering the three controllers. An automatic speed change profile was designed and implemented on the leader car, consisting of a series of speed changes in which the maximum acceleration and deceleration were $1 \mathrm{~m} / \mathrm{s}^{2}$. The reason for using this value is because the vehicles were factory-limited in the acceleration response so the controllers can be compared in the same conditions without saturating the system. The three controllers were run on the follower vehicle, driving behind the leader vehicle maintaining this speed profile.

The enhanced version of the IDM system presented in [6] was implemented, although merging vehicles from adjacent lanes are not considered in this experiment. The IDM essentially defined an acceleration target command that is generated by the following function

$$
a_{I D M}=a\left[1-\left(\frac{v}{v_{0}}\right)^{\delta}-\left(\frac{s_{0}+v T+\frac{v \Delta v}{2 \sqrt{a b}}}{s}\right)\right]
$$

where $v$ represents the current vehicle speed, $v_{0}$ the desired speed in free-flow traffic conditions (set at $120 \mathrm{~km} / \mathrm{h}$ ), $s$ the bumper-to-bumper inter-vehicle clearance, $s_{0}$ the vehicle-vehicle clearance in stand-still situations (set at 0 meters because these tests were carried out on highways at speeds higher than 


\begin{tabular}{cc} 
Parameter & Value \\
\hline \hline desired speed $v_{0}$ & $33.3 \mathrm{~m} / \mathrm{s}$ \\
free acceleration exponent $\delta$ & 4 \\
desired time gap $T$ & $1.1 \mathrm{~s}$ \\
jam distance $s_{0}$ & $0 \mathrm{~m}$ \\
maximum acceleration $a$ & $1 \mathrm{~m} / \mathrm{s}^{2}$ \\
desired deceleration $b$ & $2 \mathrm{~m} / \mathrm{s}^{2}$ \\
\hline
\end{tabular}

Table 1: IDM parameters for M56s experimental vehicles

$25 \mathrm{~m} / \mathrm{s}$ ), $T$ the minimum steady-state time gap (set at 1.1 seconds, which is a suitable value for commercial ACC systems), $a$ the maximum acceleration (set at $1 \mathrm{~m} / \mathrm{s}^{2}$ in these vehicles by the manufacturer) and $b$ the desired deceleration (set at $2 \mathrm{~m} / \mathrm{s}^{2}$ which is considered as a proper deceleration level for comfortable car-following behavior [10]). Table 1 shows the parameters used for the IDM model implemented on the experimental vehicle.

The controller was initially tested using equation (1) but this produced an unrealistic behavior. According to the IDM website [28], it needed to be updated to limit the last term in the equation to prevent it from taking negative values. Equation (1) was re-defined as

$$
a_{I D M}=a\left[1-\left(\frac{v}{v_{0}}\right)^{\delta}-\left(\frac{s_{0}+\max \left[0, v T+\frac{v \Delta v}{2 \sqrt{a b}}\right]}{s}\right)\right]
$$

The factory ACC system and the CACC controller were also tested using the same speed profile for the leading vehicle, which consisted of the following series of speed changes. In the first cycle, the vehicle drives at $25.5 \mathrm{~m} / \mathrm{s}$ for 10 seconds; then, it accelerates at a constant acceleration of $(1 / 80) g$ to reach $29.5 \mathrm{~m} / \mathrm{s}$ speed. The speed remains constant for 10 seconds, before the vehicle starts to decelerate at a constant rate of $(1 / 80) g$ to come back to a constant speed of $25.5 \mathrm{~m} / \mathrm{s}$ for 10 seconds. The second cycle repeats the same acceleration and deceleration curve with a constant acceleration of $(1 / 40) g$ and 15 seconds of driving at a constant speed at the top and bottom of each acceleration or deceleration event. The third and fourth cycles repeat the same pattern at $(1 / 20) g$ and $(1 / 10) g$ with 20 seconds of driving at a constant speed between acceleration and deceleration events. Figure 2 presents the results for the three controllers for the same speed changes. The top graph shows the speed of the leading car (solid green line) and the behavior of 
the following car when the vehicle is controlled by the factory ACC (solid red line), the CACC (solid black line) or the IDM (solid blue line). The next one depicts the acceleration response of the vehicles for these speed changes. The following graph plots the time gap error of each controller (gap reference for CACC is 0.6 seconds, while 1.1 seconds is used as reference gap for the factory ACC and the IDM). The bottom graph shows the inter-vehicle clearance distances during the three tests.

The leader vehicle is manually driven until the speed profile is activated. This transition results in initial condition recovery oscillations in the controllers during the first 20 seconds. After that, one can compare the behavior of the three controllers. For the factory ACC system, the delay in its response causes a smooth overshoot on each acceleration phase. The higher the acceleration, the bigger the overshoot. This delay can also be appreciated in the acceleration plot. The time gap error plot gives an idea about how the car-following system behaves. For the ACC, this value is mainly under 0.1 seconds, reflecting good performance. A positive error indicates that the real time gap is higher than the desired one which indicates that the controller was designed in a conservative way giving priority to the passengers safety and avoiding gaps shorter than the set point. Finally, the inter-vehicle clearance plot indicates the visual perception the driver is likely to have of the car-following performance (accuracy) of each controller. Each speed change cycle is equal to $4 \mathrm{~m} / \mathrm{s}$ so the vehicle clearance is varying between 30 and 35 meters, which gives the driver the feeling that the vehicle is engaged to the leading one, reacting to its speed changes.

For the CACC controller, the delay is drastically reduced in the speed response-which gives the driver the feeling that the vehicle responds promptly to any speed change by the leading car-and the overshoots are eliminated. Consequently, the acceleration response is similar to that of the leading vehicle. The excellent tracking of the inter-vehicle time gap is also evident, with the time gap error of almost zero during the entire test. Finally, the reduction in the time gap from 1.1 seconds to 0.6 seconds causes the inter-vehicle clearance to vary from 15 to 18 meters.

For the IDM, there is a significant delay in the response to speed changes by the leading car. It can be clearly appreciated in the last acceleration cycle, when the CACC controller responds almost at the same time as the leader, then the ACC controller responds with a significant delay with respect to the CACC and, finally, the IDM responds even slower than the ACC controller. There is a minimum undershoot in each of the speed changes. Consequently, 

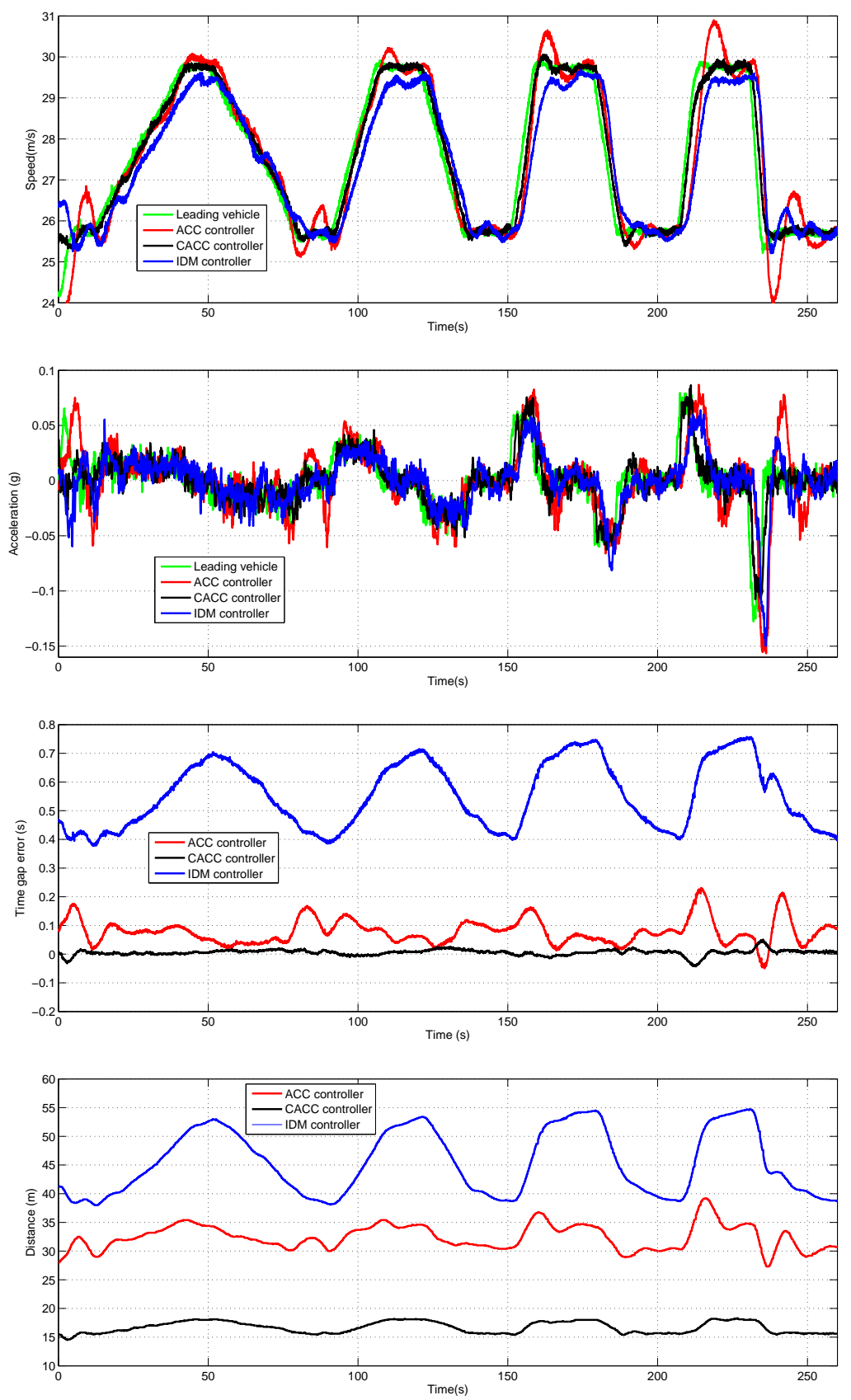

Figure 2: Comparison among car-following policies using CACC, ACC and IDM controllers experimentally tested on the M56s on public roads 
the acceleration response is pretty smooth during most of the test except for the last deceleration (when the leading vehicle brakes at $1 \mathrm{~m} / \mathrm{s}^{2}$ ). For this last deceleration maneuver, it is also noteworthy that both the ACC controller and IDM are braking harder than the leading vehicle; however, the CACC controller is braking more gently than the leading vehicle with an excellent time-gap error. The troubling behavior of the IDM is mainly reflected in the time-gap error plot. The error never goes close to zero and, additionally, it seems that there are two different time-gap references: one for the lowest speed, with a fixed gap error around 0.4 seconds; and a different one for the highest speed, with an error around 0.7 seconds.Then, the inter-vehicle clearance varies from 38 to 53 meters, so the driver perceives that the vehicle is not actively following the leader. This large gap variation raises questions about the driver acceptance of the IDM as an automatic car-following control strategy. Although IDM+ or IIDM models could improve this behavior (because they include specific modifications to deal with this time-gap error), the big delay introduced by the on-board sensors and the undershoot that is detected in the last braking maneuver would be amplified when ACCequipped vehicles (using the IDM+ or IIDM systems) drive consecutively. This result suggests that when ACC market penetration increases, it will not improve traffic flow, but will rather cause it to become less stable.

Using the experimental results obtained from the factory ACC controller and the newly developed CACC controller, an analysis of these car-following systems is next developed based on the vehicle speed responses. The next section details this analysis for each of these car-following policies.

\section{Understanding ACC and CACC car-following behavior}

This section describes the car-following behavior from the speed response perspective for the CACC and ACC vehicles. The analysis is based on test results from the same vehicles equipped with both controllers. The models defined here can be used to fill the gap between experimental results on a small number of vehicles and a microscopic simulation of many vehicles, which can be based on these simple models that incorporate accurate empirical approximations to the vehicle behavior.

To this end, the same speed profile was applied as the input to both the ACC and CACC controller, to measure the effects of speed changes with four consecutive vehicles. The goal is to get a realistic idea about how a string of vehicles works considering the effect that the leading vehicle speed 


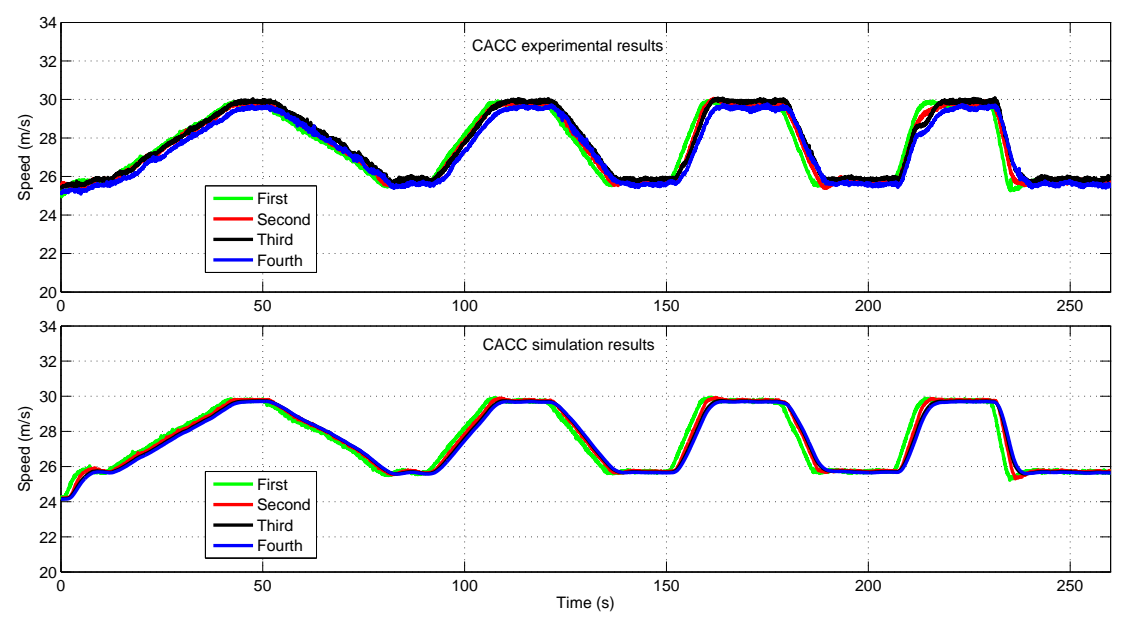

Figure 3: Comparison between simulation and experimental speed results for a CACC controller using four speed changes with different acceleration rates

change has on the second, third and fourth car for each controller. The tests were carried out on public roads with non-equipped vehicles driving in the adjacent lanes, so the magnitude and severity of the speed changes had to be strictly bounded for safety reasons.

\section{1. $C A C C$}

For the CACC controller, speed and acceleration responses for the second, third and fourth vehicles are depicted in the top graphs of Fig. 3 and 4 respectively. The good behavior of the four consecutive CACC vehicles can be appreciated, taking good advantage of the preceding vehicles' information thanks to V2V communications. The speed responses are pretty similar for all the vehicles, and they can be approximated by a first-order lag function. The System Identification Toolbox from Matlab was used for adjusting the first-order lag model parameters to achieve the best model fit. Different transfer functions, depending on the position of the vehicle, were developed. These transfer functions are presented in Table 2 where $v_{1}, v_{2}, v_{3}$ and $v_{4}$ represent the speed of the first, second, third and fourth vehicle respectively.

The real speed of the first vehicle was used to stimulate the responses of the second, third and fourth vehicles using the first-order lag transfer functions. Simulation results for speed and acceleration are shown in the bottom graph of Fig. 3 and 4 respectively. One can appreciate how they perfectly fit (over 95\%) the behavior of the real CACC vehicles validating 


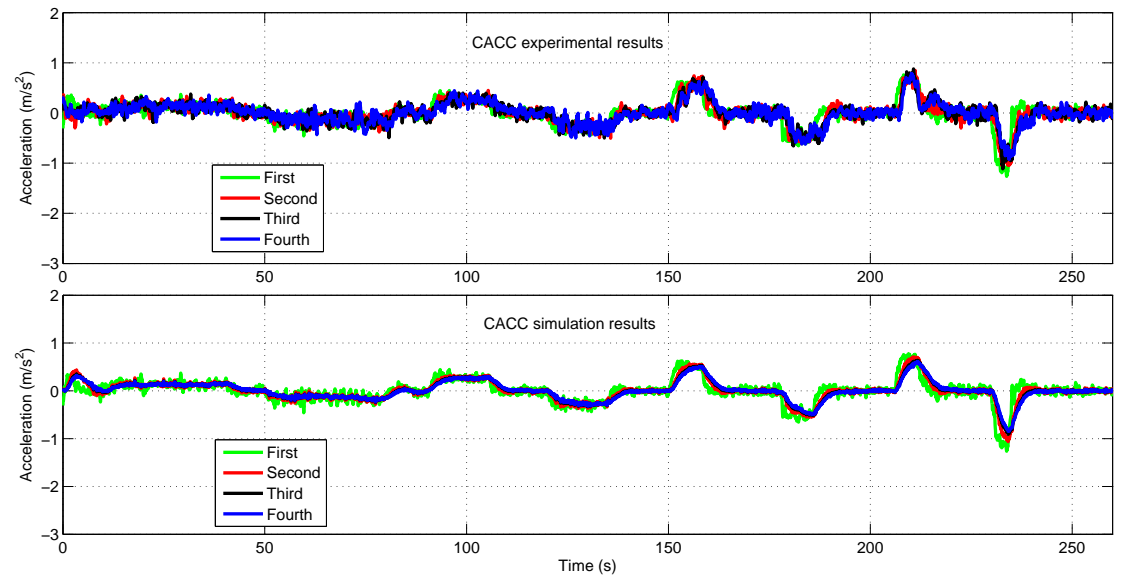

Figure 4: Comparison between simulation and experimental acceleration results for a CACC controller using four speed changes with different acceleration rates

$$
\begin{array}{ll}
\text { Second vehicle } & \frac{v_{2}}{v_{1}}=\frac{1}{1.31 s+1} \\
\text { Third vehicle } & \frac{v_{3}}{v_{1}}=\frac{1}{2.11 s+1}
\end{array}
$$$$
\text { Fourth vehicle } \quad \frac{v_{4}}{v_{1}}=\frac{1}{2.47 s+1}
$$

Table 2: CACC car-following models for the second, third and fourth vehicle 


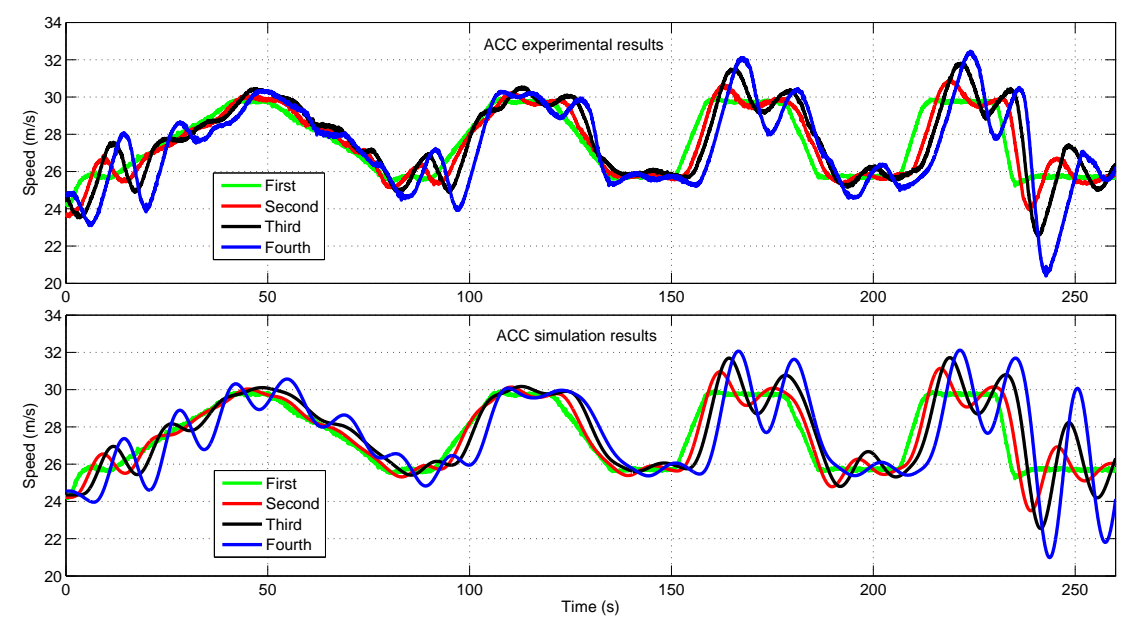

Figure 5: Comparison between simulated and experimental speed results for ACC controller using four speed changes with different acceleration rates

the proposed transfer functions. These results indicate that a simple modelrepresenting a first-order lag response-can be used to model the previously developed CACC controller [11].

\section{2. $A C C$}

The factory ACC system was also tested under the same real conditions and using the same speed profile as the CACC controller. The top graphs of Fig. 5 and 6 show the speed and acceleration respectively of the four ACC vehicles. As with the two vehicles, the initial speed of the leading vehicle before starting the speed profile is governed by a human driver so the oscillations during the first 30 seconds are for recovery of the initial condition offsets. After that, one can appreciate how the other vehicles are following the leading vehicle speed changes. As with the two vehicle results reported in Section 2.1, the higher the leading vehicle's acceleration, the higher the overshoot. That overshoot is also propagated to the rest of the vehicles so it is even larger in the third car with respect to the second car and larger again in the fourth car with respect to the third one.

This experimental result shows for the first time that the theoretical results previously presented in the literature [21, 24], where car-following policies based only on predecessor information were used for ACC controllers, are inherently unstable when applied to real vehicles. These results are contrary to the string stable results obtained with the IDM for vehicle platoon 


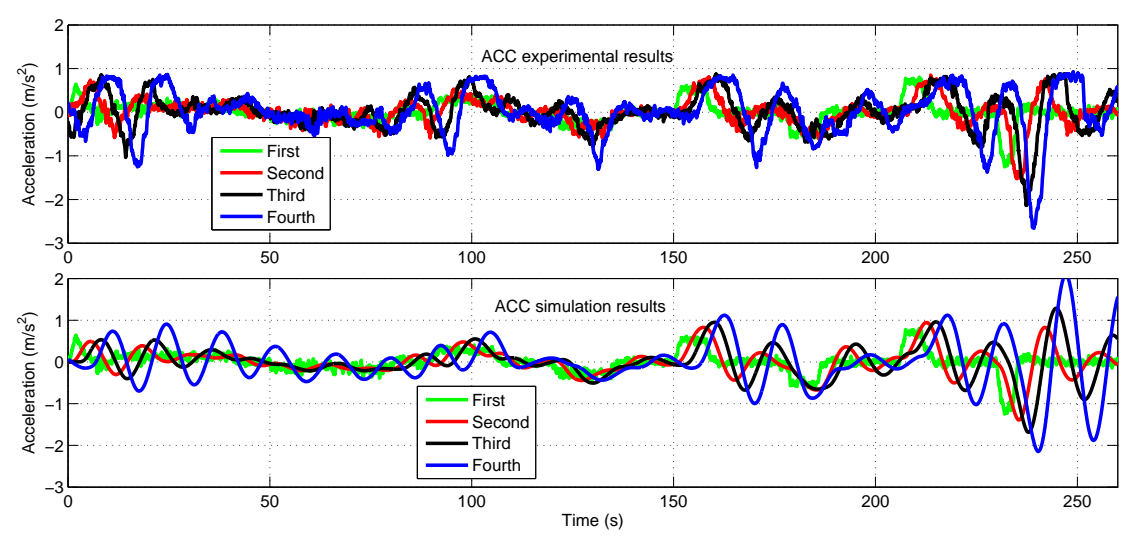

Figure 6: Comparison between simulated and experimental acceleration results for ACC controller using four speed changes with different acceleration rates

formation presented in $[7,6]$ and to the favorable traffic flow results obtained using even low ACC market penetration. The responses of the test vehicles demonstrate a considerable instability, which is amplified downstream. The difference between these results and the theoretical model results arises because the theoretical model does not represent the response delays associated with ranging sensor signal processing and vehicle actuation.

It is worth noting that this factory ACC system was designed with serious consideration of string stability, unlike many other ACC systems, and is actually more stable than some other commercially available systems. However, even its stability enhancing design features are not able to overcome the challenges associated with the lack of preview information available about the motions of the vehicles ahead of the immediately preceding vehicle.

Car-following behavior for the four ACC cars was analyzed for each car related to the leading vehicle. The System Identification Toolbox from Matlab was again used for finding the proper transfer function-second order with time delay-that represents the vehicle behavior. Table 3 shows the second-order transfer functions with time-delay that model each of the vehicles (goodness of fit over $83 \%$ for all the vehicles). Time delay plays a key role in understanding the difference between $\mathrm{CACC}$, where the vehicles receive information from all the preceding ones, and ACC, where a predecessor-only car-following policy means that the larger the number of consecutive ACC vehicles, the longer the delay.

The real speed and acceleration profiles of the first vehicle were used to 


$$
\begin{array}{ll}
\text { Second vehicle } & \frac{v_{2}}{v_{1}}=\frac{0.23}{s^{2}+0.4 s+0.23} e^{0 s} \\
\hline \text { Third vehicle } & \frac{v_{3}}{v_{1}}=\frac{0.2}{s^{2}+0.2 s+0.2} e^{-2.23 s} \\
\hline \text { Fourth vehicle } & \frac{v_{4}}{v_{1}}=\frac{0.2}{s^{2}+0.05 s+0.2} e^{-5.02 s}
\end{array}
$$

Table 3: ACC car-following models for the second, third and fourth consecutive vehicles

stimulate the responses of the second, third and fourth vehicle using the second-order transfer functions. Simulation results are shown in the bottom graphs of Fig. 5 and 6 . The larger the position of the vehicle in the platoon, the larger the disturbances. The unstable behavior of the ACC system is especially reflected at the last vehicle, making it difficult to obtain a secondorder model that fits the vehicle behavior accurately.

\section{ACC and CACC vehicle models}

The first- and second-order transfer functions clearly show the differences between ACC and CACC car-following responses for multiple consecutive vehicles. However, these open-loop responses cannot be used as car-following models. A small drift or bias can be introduced at each simulation step that cannot be recovered without closing the loop. To this end, two different models are developed: 1) a simplification of the CACC controller to fit the real vehicle behavior in a proper way is used for the CACC model; and 2) an improvement of the previous model presented in [26] based on the experimental results is used for the ACC modeling. Note that both models are limited to a maximum acceleration of $1 \mathrm{~m} / \mathrm{s}^{2}$ and a maximum deceleration of $2.8 \mathrm{~m} / \mathrm{s}^{2}$ according to the real vehicles' limitations.

\subsection{CACC vehicle model}

For modeling the CACC vehicle behavior, a simplification of the controller implemented in the production cars [11] is applied. The gap error of the $\mathrm{k}$-th consecutive vehicle $\left(e_{k}\right)$ can be determined as 


$$
e_{k}=x_{k-1}-x_{k}-t_{h w} v_{k}
$$

where $x_{k-1}$ is the current position of the preceding vehicle, $x_{k}$ and $v_{k}$ are the current position and speed of the subject vehicle respectively, and $t_{h w}$ is the current time-gap setting. The design objective for a gap regulation controller is to minimize this gap error, obtaining a constant time-gap following policy. For modeling purposes, this error and its derivative are used for determining vehicle speed on each control cycle. It is calculated following the next equation (4)

$$
v_{k}=v_{k p r e v}+k_{p} e_{k}+k_{d} \dot{e}_{k}
$$

where $v_{\text {kprev }}$ is the speed of the subject vehicle in the previous iteration, and the gains $k_{p}$ and $k_{d}$ trying to adjust the time-gap error with respect to the preceding vehicle. The same values that were determined during the experimental tests are used $\left(k_{p}=0.45\right.$ and $\left.k_{d}=0.25\right)$.

This speed function properly matches the real behavior of the vehicles $(\mathrm{RMSE}=0.1046 \mathrm{~m} / \mathrm{s})$. For its validation, the speed profile of the first real CACC vehicle is used as input for the subject vehicle model. Figure 7 shows the comparison between the second real vehicle and the second vehicle using the CACC model. The top graph depicts the speed responses of both vehicles, the middle graph plots the speed error of the real and simulated vehicle, and the bottom graph represents the distance with respect to the leading vehicle. One can appreciate how the speed of the simulated vehicle responds in the same way as the real vehicle. The time-gap adjustment is done in a similar way, which is reflected in the inter-vehicle clearance. The behavior of the speed function for the CACC model demonstrates that it is a simple and realistic way of simulating two consecutive CACC vehicles.

For the rest of the vehicles, we have used the same speed function for modeling their behavior. Figure 8 shows a comparison between the real and simulated speed response of both the third vehicle (top graph) and the fourth vehicle (bottom graph). Their responses are completely consistent with the first-order model function introduced in the previous section and with the real behavior of the vehicles (RMSE values are $0.2034 \mathrm{~m} / \mathrm{s}$ and $0.2567 \mathrm{~m} / \mathrm{s}$ respectively). 

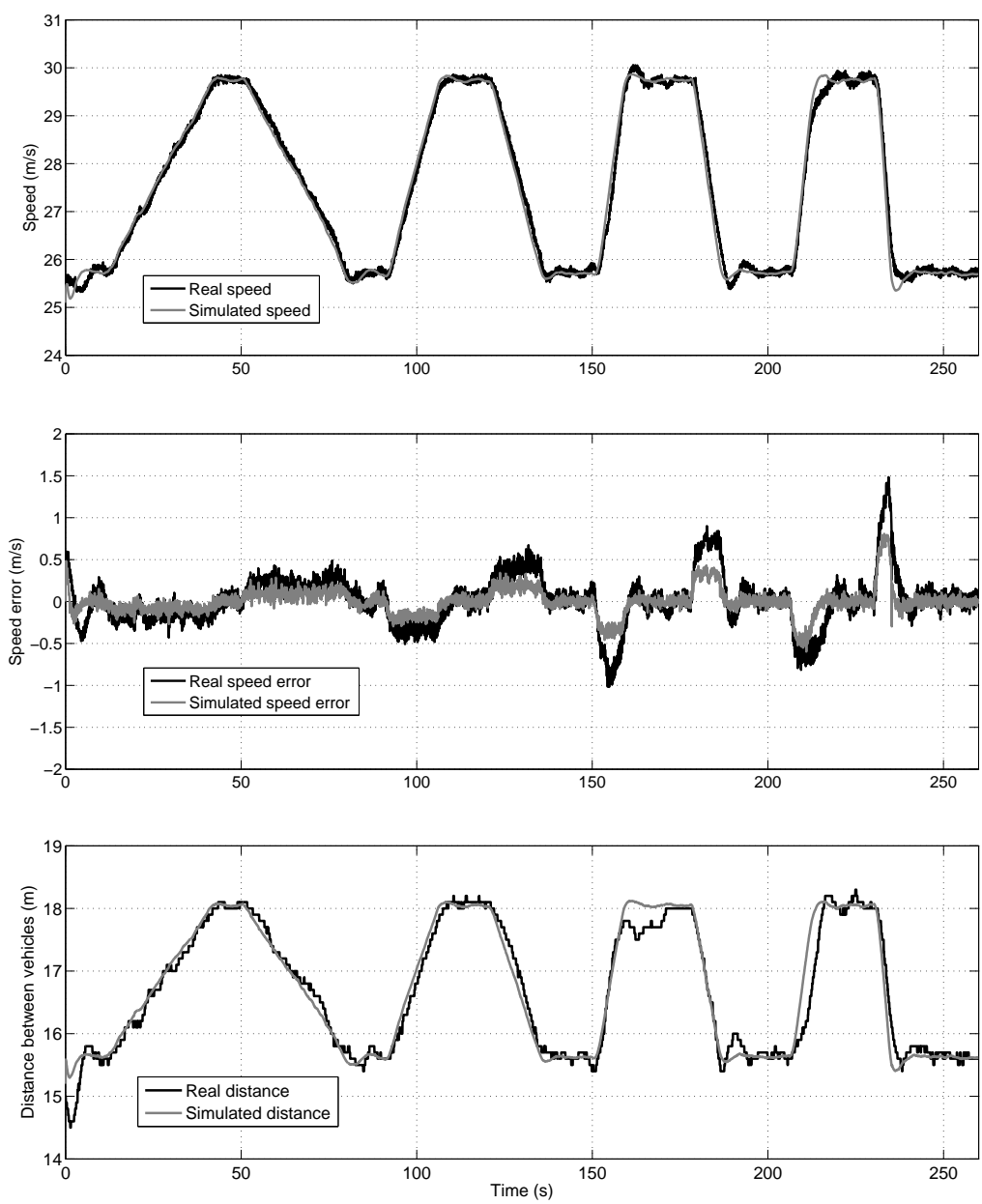

Figure 7: Comparison between real and simulated results for two consecutive CACC vehicles 

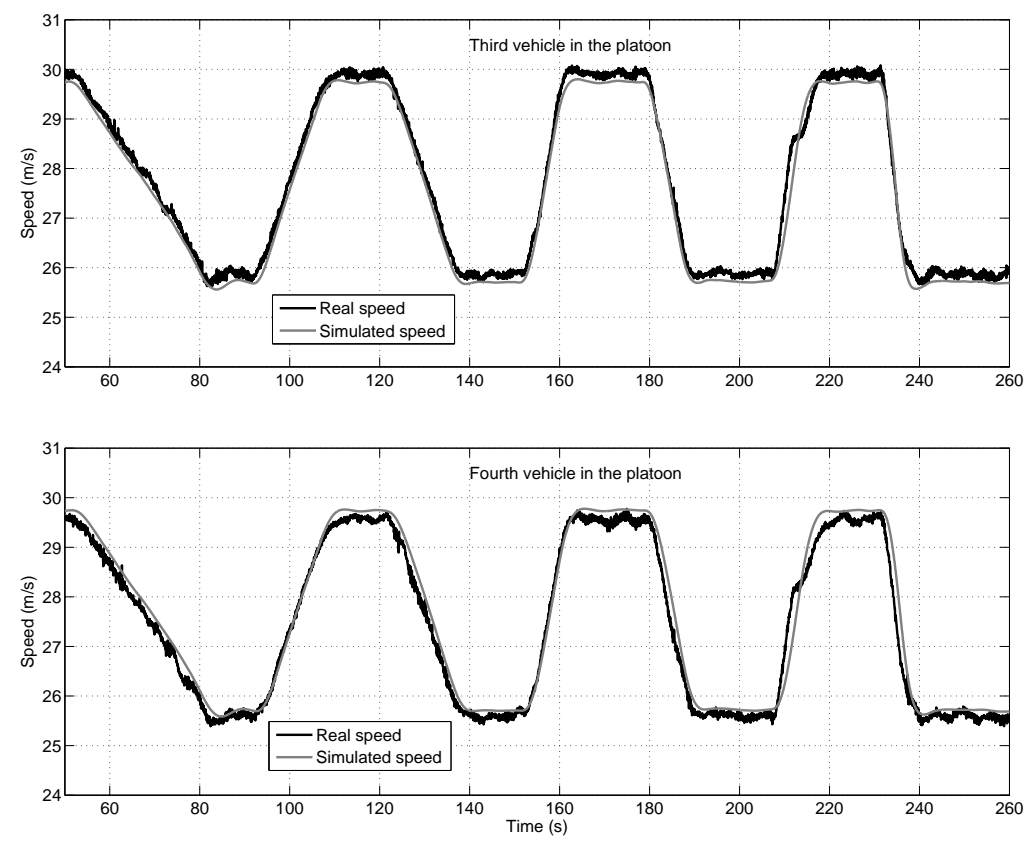

Figure 8: Comparison between real and simulated results for the third and fourth CACC vehicles 


\subsection{ACC vehicle model}

The production controller is based on several control blocks that deal with specific traffic circumstances so it is not straightforward to move the real controller into a simulation environment. Vehicle acceleration was modeled based on the distance and speed errors, according to the next equation (5)

$$
a_{k}=k_{1}\left(x_{k-1}-x_{k}-t_{h w} v_{k}\right)+k_{2}\left(v_{k-1}-v_{k}\right)
$$

where $a_{k}$ represents the acceleration of the $\mathrm{k}$-th vehicle; $x_{k-1}$ and $x_{k}$ indicate the current position of the preceding and the subject vehicle respectively; $v_{k-1}$ and $v_{k}$ represent the current speed of the preceding and the subject vehicle respectively; $t_{h w}$ is the current time-gap setting; and $k_{1}$ and $k_{2}$ are the gains on both the positioning and speed errors respectively. For determining the $k_{1}$ and $k_{2}$ gain values, an optimization criterion based on the integral absolute error (IAE) is chosen. IAE is defined as

$$
I A E=\int_{0}^{T}\left(\left|v_{\text {real }}-v_{\text {simulated }}\right|\right) d t
$$

where $v_{\text {real }}$ is the speed of the real vehicle during the four-car CACC test and $v_{\text {simulated }}$ is the speed of the same vehicle for the simulated CACC using the same speed profile. The reason for not including position error in the optimization function is the result obtained during the test. Although a constant-time-gap policy is used, the complex logic underlying the controller also considers additional parameters which results in gap variations. Bearing this in mind, an effective way to determine the gain values $k_{1}$ and $k_{2}$ for the acceleration response is minimizing the speed difference between the real and simulated results. The minimum IAE was obtained for $k_{1}=0.23 \mathrm{~s}^{-2}$ and $k_{2}=0.07 \mathrm{~s}^{-1}$.

Figure 9 shows a comparison between the real and simulated responses for the second vehicle. The top graph depicts vehicle speed, the middle graph plots speed error and the bottom graph shows distance error between vehicles. One can appreciate how well the vehicle speed obtained from the model fits the real speed from the experimental data $(\mathrm{RMSE}=0.2984 \mathrm{~m} / \mathrm{s})$. Speed and distance error results also show how the vehicle is following those errors in a proper way-considering real vehicle errors.

Figure 10 shows the behavior of the third and fourth ACC vehicles using the same model (RMSE values are $0.5149 \mathrm{~m} / \mathrm{s}$ and $0.8226 \mathrm{~m} / \mathrm{s}$ respectively). As can be appreciated in the second-order transfer function for the vehicle 

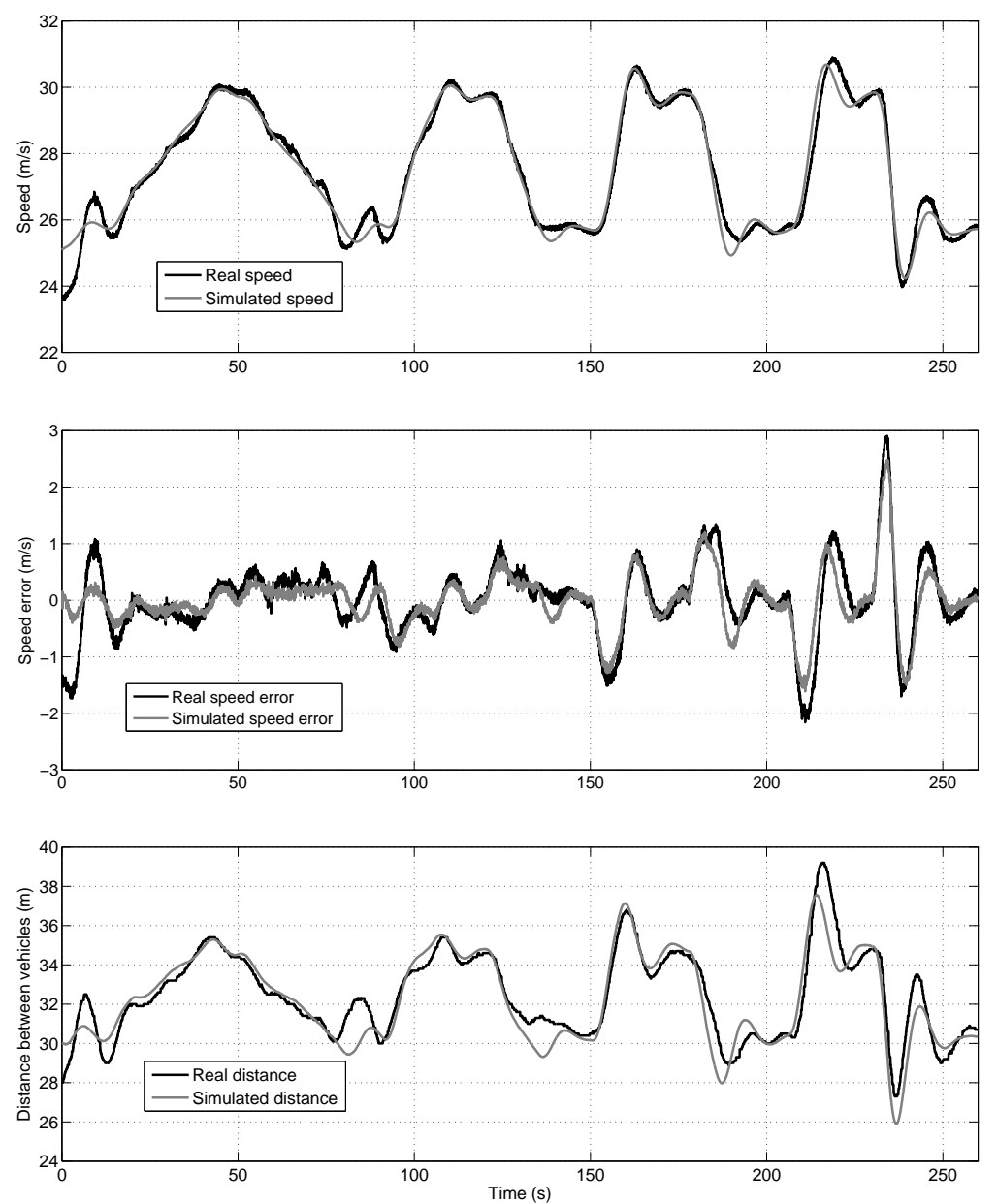

Figure 9: Comparison between real and simulated results using two consecutive ACC vehicles 

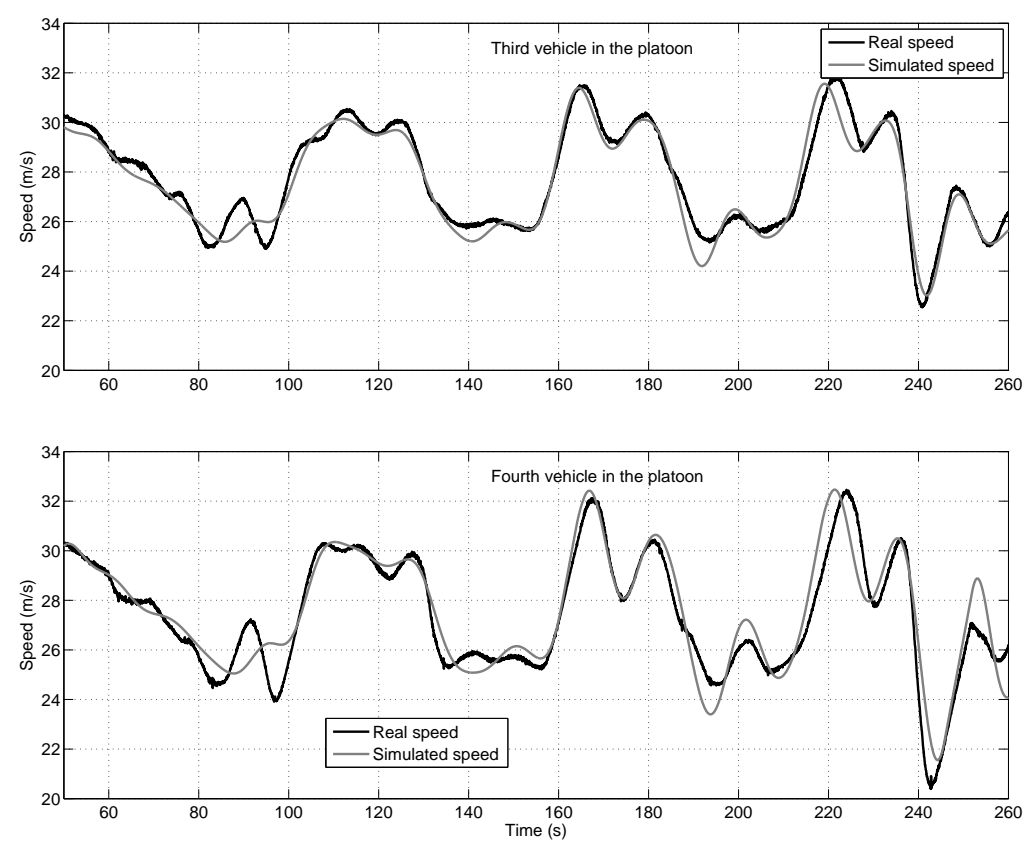

Figure 10: Comparison between real and simulated results for the third and fourth ACC vehicle

speed presented in the previous section, the time delay from one vehicle to the next is pretty similar so the same vehicle model was used for each vehicle, using its preceding vehicle to determine the position and speed errors. Note that although the model was tested for four vehicles, it could potentially be extended to an unlimited number of vehicles. However, considering the feedback from drivers who experienced the controller in the fourth vehicle, a human driver would be likely to disengage the controller since the behavior is clearly uncomfortable and considerably worse than a human driver is likely to permit without intervening. It is also remarkable to comment how the basic ACC model car following fits well even for the last vehicle.

Recall that this model is a description of the performance of the commercially available ACC system, which has been carefully designed to give smooth performance that will be acceptable to drivers when used in a twocar vehicle following scenario. Changing the control gains to improve string stability may have adverse effects on other aspects of car following behavior, impairing driver acceptability, so it should not be undertaken lightly. Additional research is needed to determine how to design ACC systems that will 
meet driver expectations in two-car following scenarios while also minimizing string instability in multi-car following scenarios.

\section{Simulation results}

This section presents some simulation results to analyze different scenarios when the number of consecutive ACC or CACC vehicles increases. Specifically, three cases are considered: 1) ten consecutive CACC vehicles; 2) five consecutive ACC vehicles; and 3) a mixed case, where the two first followers are ACC-equipped and the next seven are CACC-equipped.

\subsection{Ten-vehicle $C A C C$ scenario}

This test shows the effect of increasing the number of consecutive CACCequipped vehicles. Using the model obtained in the previous section, six additional vehicles are simulated to assess how longer strings behave. The top of the Figure 11 depicts vehicle responses to the speed changes on the part of the leading vehicle. For the sake of clarity, the fifth, seventh and ninth vehicle responses are not included in the graphic. The speed of the leading vehicle was obtained from the road tests. One can appreciate how all the vehicles perfectly follow the leading vehicle speed changes without amplifying its oscillations downstream. This result corresponds well with the experimental results shown for the four-vehicle test in Figure 3. It gives an idea about the ability of CACC to improve traffic flow stability and highway capacity. The middle and bottom plot show clearance distances between vehicles and time gap values during the test.

\subsection{Five-vehicle $A C C$ scenario}

Experimental results showed the amplification downstream when the leading vehicle speed changes in ACC formations. To study the effects when the number of vehicles increases, a simulation scenario with five consecutive vehicles is presented in Fig. 12. The top graph plots vehicle speeds; the middle graph depicts clearance distance between vehicles; and the bottom graph shows the time gap of each vehicle during the experiment. The most undesirable behavior appears in the last braking maneuver around second 240. A deceleration of $1 \mathrm{~m} / \mathrm{s}^{2}$ from 30 to $26 \mathrm{~m} / \mathrm{s}$ by the leading vehicle causes the last vehicle to brake to $20 \mathrm{~m} / \mathrm{s}$. It is also noteworthy that the delay introduced by each vehicle can cause the vehicles to get out of phase with each other, to the extent that when the leading vehicle begins to accelerate, the last vehicle in 

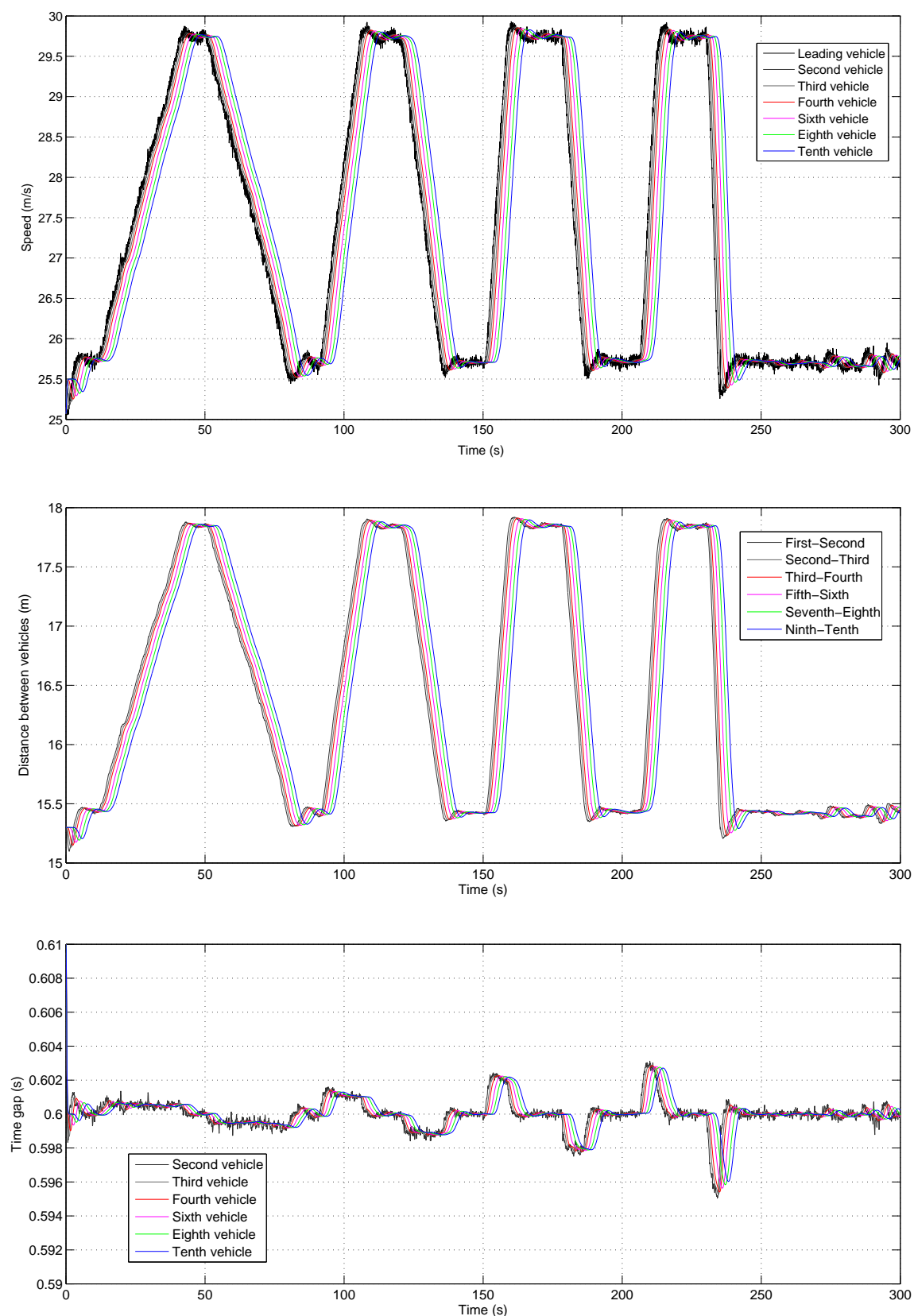

Figure 11: Simulation results for ten consecutive CACC vehicles 
the platoon is braking, as occurs around second 210. These oscillations were so severe that the test drivers rated them unacceptable for normal use. Even with the gentle speed change profile of the leading vehicle, if more consecutive ACC-equipped vehicles are included and assuming drivers keep their ACC running during the whole test, a full stop would occur at the tenth vehicle. This indicates the instability that ACC-equipped vehicles can introduce in traffic flow at high market penetration ranges, reducing highway capacity.

\subsection{Scenario with mixture of CACC-equipped and ACC-equipped vehicles}

The last simulation experiment consists of ten vehicles, where the second and third vehicles are ACC-equipped (the third vehicle is also equipped with V2V communications) and the next seven vehicles are CACC-equipped. The top graph of Figure 13 shows the speed of the vehicles during the simulation. The same speed profile as used in the two previous tests is used for the leading vehicle. One can appreciate how the second and third vehicles amplify the speed changes of the leading vehicle. Then, from the fourth vehicle, the oscillations are considerably reduced. The bottom part of Figure 13 plots the behavior of the seven CACC-equipped vehicles (removing the leader and the second vehicle). The unstable behavior of the third vehicle (the leader for the next seven CACC-equipped vehicles) can be clearly seen, and then the rest of the vehicles considerably reduce its oscillations, improving traffic flow and stability.

\section{Concluding remarks}

This paper has reported two primary findings. First, it shows the comparison in car following performance among three different controllers, a production ACC, the IDM model that has been widely used for ACC car-following simulations and a newly developed CACC controller, using test results on production vehicles in order to evaluate their performance in realistic traffic circumstances. Second, it applies the experimental results to derive simple car-following models that can be used to represent the production ACC and the new CACC in microscopic simulations of their impacts on traffic flow dynamics.

The comparison among the different controllers shows the IDM model producing smooth car-following behavior, but with very slow response and large clearance gap variations. This comparison also demonstrates that string stability is not achievable for multiple consecutive vehicles using ACC when 

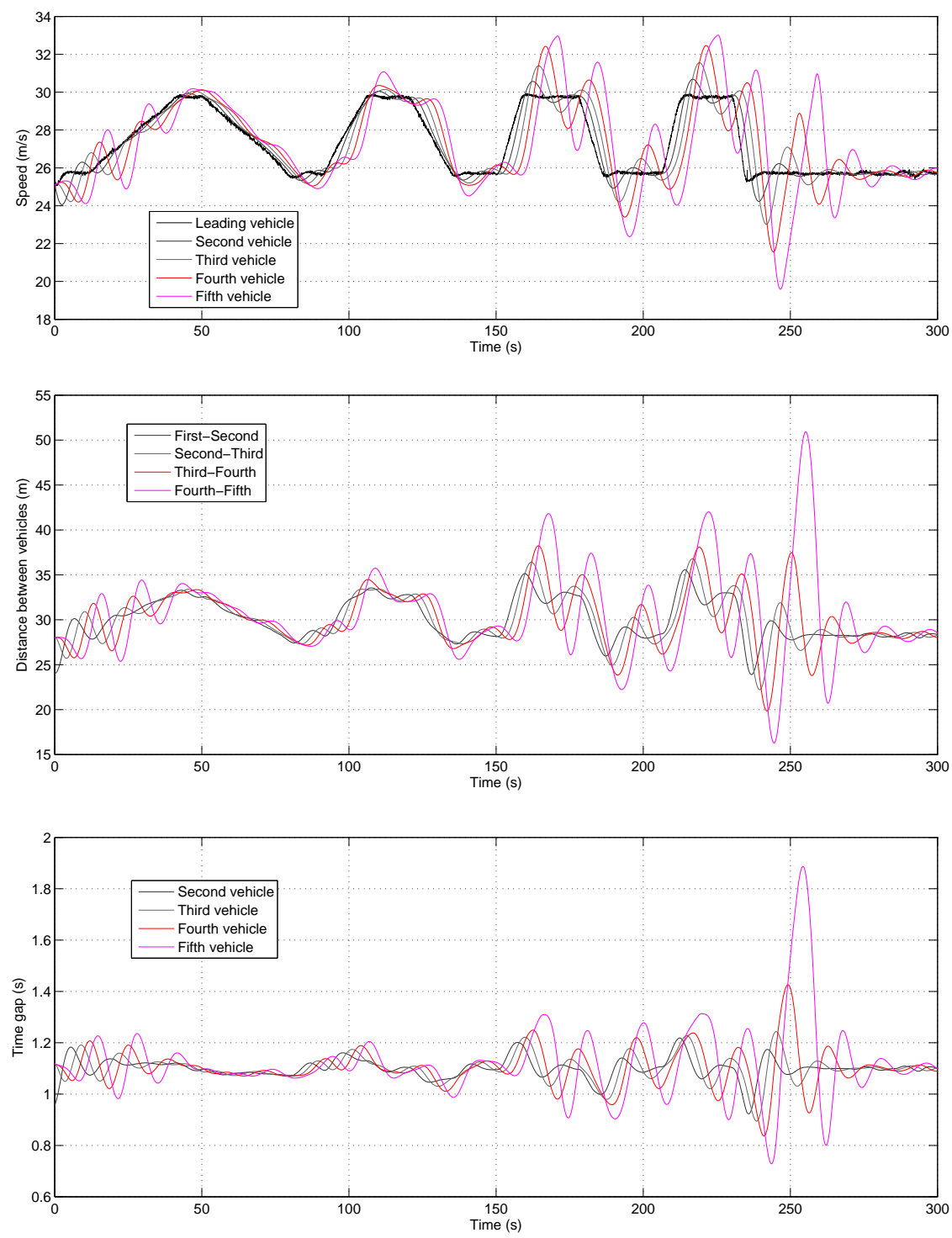

Figure 12: Simulation results for five consecutive ACC vehicles 


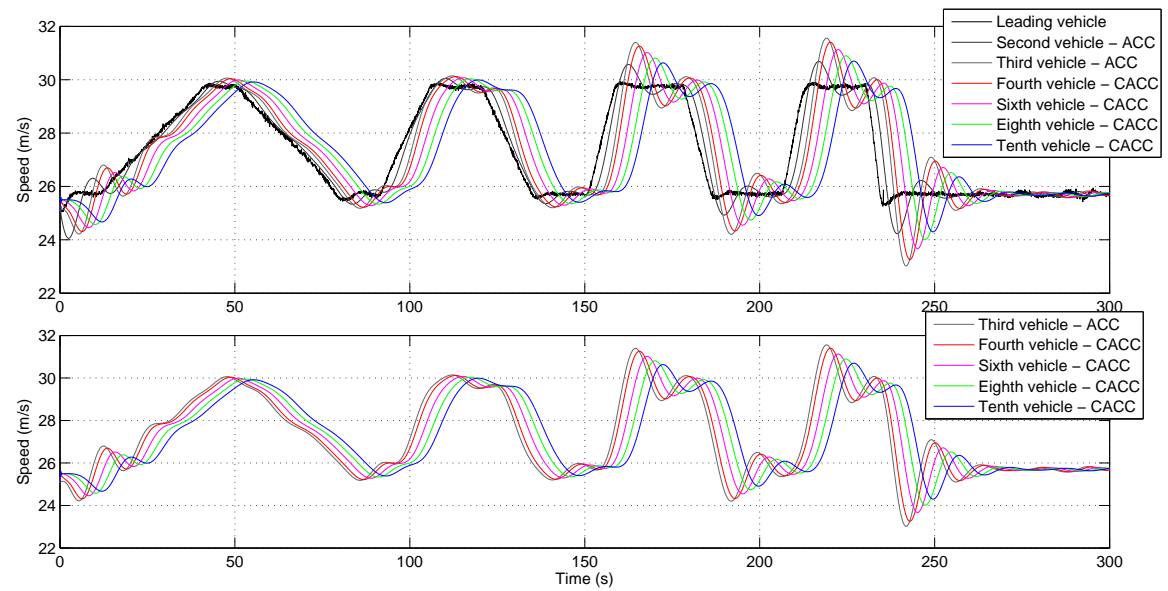

Figure 13: Simulation results for ten vehicles with a mix of ACC followed by CACC

the leading vehicle speed varies, even under relatively mild speed variations. Although the speed changes smoothly, the responses include a large enough delay to cause an unstable response of the following vehicles. This instability is solved by adding V2V communications, leading to the CACC controller. Experimental results demonstrate that $\mathrm{CACC}$ is a suitable candidate to produce a significant improvement in highway capacity and traffic flow stability.

The newly developed models for both ACC and CACC systems match the experimental results obtained from the production vehicle road tests very closely. A comparison between speed and clearance distance errors between the real and simulated vehicles is included for both controllers, indicating the accuracy of the proposed models.

Next research steps are focused on two goals: on one hand, to implement the proposed models in a microsimulation platform to evaluate their effects on traffic flow for different market penetration ranges; on the other hand, to improve the proposed models toward a more realistic behavior including cut-in and cut-out responses from unequipped vehicles using experimental results obtained from our four CACC vehicles [11].

\section{Acknowledgment}

The PATH work on this project was sponsored by Nissan Motor Co. Ltd. V. Milans wants to especially thanks to the ME/Fulbright program and the 
Center for Automation and Robotics (CAR,UPM-CSIC) for its support in the development of this work.

\section{References}

[1] M. Brackstone \& M. McDonald (1999). 'Car-following: a historical review'. Transportation Research Part F: Psychology and Behaviour 2(4):181-196.

[2] F. Bu, et al. (2010). 'Design and field testing of a Cooperative Adaptive Cruise Control system'. In Proc. American Control Conf. (ACC), pp. 4616-4621.

[3] A. Geiger, et al. (2012). 'Team AnnieWAY's Entry to the 2011 Grand Cooperative Driving Challenge'. IEEE Transactions on Intelligent Transportation Systems 13(3):1008-1017.

[4] L. Guvenç, et al. (2012). 'Cooperative Adaptive Cruise Control Implementation of Team Mekar at the Grand Cooperative Driving Challenge'. IEEE Transactions on Intelligent Transportation Systems 13(3):10621074.

[5] K. Jerath \& S. Brennan (2012). 'Analytical Prediction of Self-Organized Traffic Jams as a Function of Increasing ACC Penetration'. IEEE Transactions on Intelligent Transportation Systems 13(4):1782-1791.

[6] A. Kesting, et al. (2010). 'Enhanced intelligent driver model to access the impact of driving strategies on traffic capacity'. Philosophical Transactions of The Royal Society A 368:4585-4605.

[7] A. Kesting, et al. (2008). 'Adaptive cruise control design for active congestion avoidance'. Transportation Research Part C: Emerging Technologies 16(6):668-683.

[8] R. Kianfar, et al. (2012). 'Design and Experimental Validation of a Cooperative Driving System in the Grand Cooperative Driving Challenge'. IEEE Transactions on Intelligent Transportation Systems 13(3):994-1007.

[9] K. Lidstrm, et al. (2012). 'A Modular CACC System Integration and Design'. IEEE Transactions on Intelligent Transportation Systems 13(3):1050-1061. 
[10] V. Milanes, et al. (2012). 'Low-Speed Longitudinal Controllers for MassProduced Cars: A Comparative Study'. IEEE Transactions on Industrial Electronics 59(1):620-628.

[11] V. Milans, et al. (2014). 'Cooperative Adaptive Cruise Control in Real Traffic Situations'. IEEE Transactions on Intelligent Transportation Systems 15(1):296-305.

[12] V. Milans, et al. (2012). 'Comparing Fuzzy and Intelligent PI Controllers in Stop-and-Go Manoeuvres'. IEEE Transactions on Control Systems Technology 20(3):770-778.

[13] S. Moon, et al. (2009). 'Design, tuning, and evaluation of a full-range adaptive cruise control system with collision avoidance'. Control Engineering Practice 17(4):442-455.

[14] J. Naranjo, et al. (2006). 'ACC+Stop\&go maneuvers with throttle and brake fuzzy control'. IEEE Transactions on Intelligent Transportation Systems 7(2):213-225.

[15] G. Naus, et al. (2010). 'Design and implementation of parameterized adaptive cruise control: An explicit model predictive control approach'. Control Engineering Practice 18:882-892.

[16] D. Ngoduy (2013). 'Instability of cooperative adaptive cruise control traffic flow: A macroscopic approach Original Research Article'. Communications in Nonlinear Science and Numerical Simulation in press:1-8.

[17] M. Nieuwenhuijze, et al. (2012). 'Cooperative Driving With a HeavyDuty Truck in Mixed Traffic: Experimental Results'. IEEE Transactions on Intelligent Transportation Systems 13(3):1026-1032.

[18] J. Ploeg, et al. (2011). 'Design and experimental evaluation of cooperative adaptive cruise control'. In Proc. 14th Int Intelligent Transportation Systems (ITSC) IEEE Conf, pp. 260-265.

[19] W. Schakel, et al. (2010). 'Effects of Cooperative Adaptive Cruise Control on Traffic Flow Stability'. In 13th IEEE Conference on Intelligent Transportation Systems, pp. 759-764. 
[20] D. Schrank, et al. (2012). 'Urban Mobility Report'. Tech. rep., Texas A\&M Transportation Institute.

[21] P. Seiler, et al. (2004). 'Disturbance propagation in vehicle strings'. IEEE Transactions on Automatic Control 49(10):1835-1842.

[22] C. Serafin (1996). 'Driver Preferences and Usability of Adjustable Distance Controls for an Adaptive Cruise Control (ACC) System'. Tech. rep., National Highway Traffic Safety Administration.

[23] A. Shaout \& M. Jarrah (1997). 'Cruise control technology review'. Computers $\&$ Electrical Engineering 23(4):259-271.

[24] E. Shaw \& J. K. Hedrick (2007). 'String Stability Analysis for Heterogeneous Vehicle Strings'. In Proc. American Control Conference, pp. $3118-3125$.

[25] S. Shladover, et al. (2010). 'Cooperative Adaptive Cruise Control: Driver Selection of Car-following Gaps'. In 17th ITS World Congress.

[26] S. Shladover, et al. (2012). 'Impacts of Cooperative Adaptive Cruise Control on Freeway Traffic Flow'. In Transportation Research Record, no. 2342, pp. 63-70.

[27] D. Swaroop, et al. (1994). 'A Comparision of Spacing and Headway Control Laws for Automatically Controlled Vehicles'. Vehicle System Dynamics: International Journal of Vehicle Mechanics and Mobility 23(1):597625 .

[28] M. Treiber (2013). 'http://www.vwi.tudresden.de/ treiber/MicroApplet/IDM.html'.

[29] M. Treiber, et al. (2000). 'Congested Traffic States in Empirical Observations and Microscopic Simulations'. Physical Review E 62:1805-1824.

[30] M. Treiber \& A. Kesting (2013). Traffic flow dynamics: Data, Models and Simulations. Springer.

[31] B. van Arem, et al. (2006). 'The Impact of Cooperative Adaptive Cruise Control on Traffic-Flow Characteristics'. IEEE Transactions on Intelligent Transportation Systems 7(4):429-436. 
[32] E. van Nunen, et al. (2012). 'Cooperative Competition for Future Mobility'. IEEE Transactions on Intelligent Transportation Systems 13(3):10181025.

[33] P. Varaiya (1993). 'Smart cars on smart roads: Problems of control'. IEEE Transactions on Automatic Control 38(2):195-207.

[34] M. Weinberger, et al. (2001). 'Adaptive cruise control field operational test-the learning phase'. JSAE Review 22(4):487-494. 